\title{
THE RANGE OF NON-SURJECTIVE CONVOLUTION OPERATORS ON BEURLING SPACES
}

\author{
by JOSÉ BONET and ANTONIO GALBIS
}

(Received 27 September, 1994)

\begin{abstract}
Let $\mu \neq 0$ be an ultradistribution of Beurling type with compact support in the space $\mathscr{E}_{\omega}^{\prime}\left(\mathbb{R}^{N}\right)$. We investigate the range of the convolution operator $T_{\mu}$ on the space of non-quasianalytic functions of Beurling type $\mathscr{E}_{\omega}\left(\mathbb{R}^{N}\right)$ associated with a weight $\omega$, in the case the operator is not surjective. It is proved that the range of $T_{\mu}$ always contains the space of real-analytic functions, and that it contains a smaller space of Beurling type $\mathscr{E}_{\sigma r}\left(\mathbb{R}^{N}\right)$ for a weight $\sigma \geq \omega$ if and only if the convolution operator is surjective on the smaller class.
\end{abstract}

Introduction. The problem of characterizing the surjectivity of convolution operators and, in particular, of partial differential operators, has interested several authors. Malgrange $[20,21]$ and Ehrenpreis $[7,8]$ gave necessary and sufficient conditions on the open subset $\Omega$ of $\mathbb{R}^{N^{\prime}}$ for the surjectivity of a partial differential operator with constant coefficients on the spaces $\mathscr{D}^{\prime}(\Omega)$ or $\mathscr{D}_{F}^{\prime}(\Omega)$ (distributions with a finite order). For a distribution $\mu \in \mathscr{C}^{\prime}\left(\mathbb{R}^{N}\right), \mu \neq 0$, Ehrenpreis [9] gave a criterion for the surjectivity of the convolution operator $T_{\mu}: C^{\infty}\left(\mathbb{R}^{N}\right) \rightarrow C^{\infty}\left(\mathbb{R}^{N}\right), f \rightarrow \mu * f$, in terms of the behavior of the Fourier transform of $\mu$. Also see Hörmander [15]. Extensions of the Ehrenpreis results to some other spaces of functions or distributions were obtained by different authors. See for instance [5], [6], [10], [12], [24], [25] and the references given in [26]. In particular, the surjective convolution operators on non-quasianalytic classes of Beurling type were characterized by Meise, Taylor and Vogt [24] (see also [4]).

In the present note we investigate the range of the non-surjective convolution operators on the non-quasianalytic classes of Beurling type $\mathscr{E}_{\omega}\left(\mathbb{R}^{N}\right)$. The problem we are interested in here can be stated as follows. Fix a weight function $\omega$ and an ultradistribution $\mu \in \mathscr{E}_{\omega}^{\prime}\left(\mathbb{R}^{N}\right)$ such that the associated convolution operator $T_{\mu}: \mathscr{E}_{\omega}\left(\mathbb{R}^{N}\right) \rightarrow \mathscr{E}_{\omega}\left(\mathbb{R}^{N}\right)$ is not surjective. We want to analyze which classes of (real-analytic or) non-quasianalytic functions are still contained in the range of $T_{\mu}$. It turns out that the class of real-analytic functions is always contained in the range of $T_{\mu}$ (Theorem 1). This is an extension of a classical result of Hörmander (e.g. [18, 16.4.5]). The study of the non-quasianalytic classes yields the following result. For two weights $\omega \leq \sigma$ (i.e. $\left.\mathscr{E}_{\sigma}\left(\mathbb{R}^{N}\right) \subset \mathscr{E}_{\omega}\left(\mathbb{R}^{N}\right)\right)$ and $\mu \in \mathscr{E}_{\omega}^{\prime}\left(\mathbb{R}^{N}\right)$, the range of the operator $T_{\mu}$ contains $\mathscr{E}_{\sigma}\left(\mathbb{R}^{N}\right)$ if and only if the operator is surjective in the smaller class. As a consequence, whenever it is possible to solve the equation $\mu * g=f$ in $\mathscr{E}_{\omega}\left(\mathbb{R}^{N}\right)$ for every $f \in \mathscr{E}_{\sigma}\left(\mathbb{R}^{N}\right)$ it is also possible to solve the same equation in $\mathscr{E}_{\sigma}\left(\mathbb{R}^{N}\right)$ for every $f \in \mathscr{E}_{\sigma}\left(\mathbb{R}^{N}\right)$. This is Theorem 2.

The proof of our main results is based on some (rather elementary) functional analytic lemmas which permit us, using Fourier-Laplace transforms, to reduce our original problem to the continuity of a division operator between two different algebras of entire functions.

The surjectivity of a convolution operator $T_{\mu}$ on $\mathscr{E}_{\omega}\left(\mathbb{R}^{N}\right)$ is equivalent to $T_{\mu}^{\prime} \mathscr{E}_{\omega}^{\prime}\left(\mathbb{R}^{N}\right)$ being a (DFS)-space. However we provide examples of non-invertible distributions 
$\mu \in \mathscr{E}^{\prime}\left(\mathbb{R}^{N}\right)$ such that $T_{\mu}^{\prime} \mathscr{E}^{\prime}\left(\mathbb{R}^{N}\right)$ is bornological, answering in the negative a question of Ehrenpreis ([9]). Our results can be applied in particular to Gevrey classes.

Our approach to the non-quasianalytic classes of Beurling type is as in [3]. We refer to [28, Chapter 8] and [11] for information concerning Fréchet-Schwartz and (DFS) (i.e. strong duals of Fréchet-Schwartz) spaces.

In the rest of the article the convolution operator $T_{\mu}$ is associated with an ultradistribution $\mu \neq 0$.

\section{Preliminaries.} satisfies:

Definimon. A continuous increasing function $\omega:[0, \infty[\rightarrow[0, \infty[$ is called a weight if it

$(\alpha)$ there exists $K \geq 1$ with $\omega(2 t) \leq K(\omega(t)+1)$ for all $t \geq 0$,

(B) $\int_{0}^{\infty} \frac{\omega(t)}{1+t^{2}}<\infty$

( $\gamma) \log \left(1+t^{2}\right)=O(\omega(t))$ as $t$ tends to $\infty$,

( $\delta) \varphi: t \rightarrow \omega\left(e^{t}\right)$ is convex.

In the examples we will also consider the following property on a weight:

$\left(\alpha^{\prime}\right) \omega(0)=0$ and $\omega(s+t) \leq \omega(s)+\omega(t)$.

For each weight $\omega$ we have $\lim _{t \rightarrow \infty} \frac{\omega(t)}{t}=0$. The Young conjugate $\varphi^{*}:[0, \infty[\rightarrow \mathbb{R}$ of $\varphi$ is defined by

$$
\varphi^{*}(s):=\sup \{s t-\varphi(t): t \geq 0\} .
$$

DEFINITION. The space of ultradifferentiable functions of Beurling type $\mathscr{E}_{\omega}\left(\mathbb{R}^{N}\right)$ associated to a weight $\omega$ is the Fréchet-Schwartz space of all functions $f \in C^{\infty}\left(\mathbb{R}^{N}\right)$ such that for each $K \subset \mathbb{R}^{N}$ compact and each $m \in \mathbb{N}$ :

$$
P_{K, m}(f):=\sup _{x \in K} \sup _{\alpha \in \mathbb{N}_{0}^{*}}\left|f^{(\alpha)}(x)\right| \exp \left(-m \varphi^{*}\left(\frac{|\alpha|}{m}\right)\right)<\infty .
$$

Examples (see for instance [23], [24]). The following functions $\omega:[0, \infty[\rightarrow[0, \infty[$ (after a suitable change on $[0, A]$ for some $A>0$ ) are the weight functions:

(1) $\omega(t)=(\log (1+t))^{s}, s \geq 1$. For $s=1$ we get $\mathscr{E}_{\omega}\left(\mathbb{R}^{N}\right)=C^{\star}\left(\mathbb{R}^{N}\right)$.

(2) $\omega(t)=\exp \left((\log (1+t))^{\alpha}\right), 0<\alpha<1$.

(3) $\omega(t)=t^{\alpha}(\log (1+t))^{\beta}, 0<\alpha<1,0 \leq \beta \leq 1$.

(4) $\omega(t)=t^{1 / s}, s>1$. In this case $\mathscr{E}_{\omega}\left(\mathbb{R}^{N}\right)=\Gamma^{(s)}\left(\mathbb{R}^{N}\right)$ is the Gevrey space of order $s$.

For $\mu \in \mathscr{E}_{\omega}^{\prime}\left(\mathbb{R}^{N}\right)$ the convolution operator $T_{\mu}: \mathscr{E}_{\omega}\left(\mathbb{R}^{N}\right) \rightarrow \mathscr{E}_{\omega}\left(\mathbb{R}^{N}\right)$ is defined by $T_{\mu}(\varphi)(x):=(\mu * \varphi)(x)=\left\langle\mu_{y}, \varphi(x-y)\right\rangle$, which is a continuous linear operator with transpose map $T_{\mu}^{\prime}: \mathscr{E}_{\omega}^{\prime}\left(\mathbb{R}^{N}\right) \rightarrow \mathscr{E}_{\omega}^{\prime}\left(\mathbb{R}^{N}\right), T_{\mu}^{\prime}(v):=\check{\mu} * v$, where $\check{\mu}(\varphi):=\mu(\check{\varphi})$ and $\check{\varphi}(x):=\varphi(-x)$.

If $\omega$ is a weight we put $p(z):=\omega(|z|)+|\operatorname{Im} z|, z \in \mathbb{C}^{N}$. Given a continuous function $p$ on $\mathbb{C}^{N}$, we let $A_{p}$ denote the (DFS)-space of entire functions

$$
A_{p}:=\left\{f \in H\left(\mathbb{C}^{N}\right): \text { there is } m \in \mathbb{N} \text { with } \sup _{z \in \mathbb{C}^{N}}|f(z)| \exp (-m p(z))<\infty\right\} .
$$


In particular for $p(z):=|z|, A_{p}$ is the space $\operatorname{Exp}\left(\mathbb{C}^{N}\right)$ of entire functions of exponential type (see [16]).

It is well known that the Fourier-Laplace transform $F: \mu \rightarrow \hat{\mu}, \hat{\mu}(z):=\left\langle\mu_{x}, e^{-i\langle x, z\rangle}\right\rangle$, is an algebra isomorphism between the convolution algebra $\left(\mathscr{E}_{\omega}^{\prime}\left(\mathbb{R}^{N}\right), *\right)$ and $A_{p}$, which is even a topological isomorphism when we endow $\mathscr{E}_{\omega}^{\prime}\left(\mathbb{R}^{N}\right)$ with the strong topology. We will identify, via Fourier-Laplace transform, the transpose map $T_{\mu}^{\prime}$ with the multiplication operator $F T_{\mu}^{\prime} F^{-1}: A_{p} \rightarrow A_{p}, f \rightarrow \hat{\tilde{\mu} f}$. Sometimes we will also identify $\mathscr{E}_{\omega}^{\prime}\left(\mathbb{R}^{N}\right)$ with $A_{p}$.

We let $E_{\mu}$ denote the set of all linear combinations of exponential solutions of the equation $\mu * u=0$, that is, solutions of the form $u(x)=P(x) e^{i(x, \xi)}$, where $\xi \in \mathbb{C}^{N}$ and $P$ is a polynomial.

Our first result describes the closure of the image of the transpose of an arbitrary convolution operator and it will be used several times in the article. The case $N=1$ is a consequence of $[19,6.13]$.

Proposition 1. Let $\mu, v \in \mathscr{C}_{\omega}^{\prime}\left(\mathbb{R}^{N}\right), \mu \neq 0$. The following conditions are equivalent:

(1) $v \in \overline{T_{\mu}^{\prime}\left(\mathscr{E}_{\omega}^{\prime}\left(\mathbb{R}^{N}\right)\right)}$

(2) $v$ is orthogonal to $E_{\mu}$

(3) $f:=\hat{v} / \hat{\tilde{\mu}}$ is an entire function on $\mathbb{C}^{N}$.

Proof. First observe that $\overline{T_{\mu}^{\prime}\left(\mathscr{E}_{\omega}^{\prime}\left(\mathbb{R}^{N}\right)\right)}$ is the orthogonal of ker $T_{\mu}$. Accordingly, (1) implies (2) is immediate, and (2) implies (3) can be obtained as in [17, Lemma 7.37] (also see [18, Lemma 16.4.2]). To complete the proof of (3) implies (1) we assume that $f:=\hat{v} / \hat{\tilde{\mu}}$ is an entire function.

We first prove that $f$ is of exponential type. In fact, the Paley-Wiener theorem implies that $\hat{v}$, and $\hat{\tilde{\mu}}$ are in $\operatorname{Exp}\left(\mathbb{C}^{N}\right)$. Let $I_{a}$ denote the ideal in the local ring $O_{a}$ which is generated by the germ $[\check{\mu}]_{a}$ of $\check{\mu}$ at $a$. Since the principal ideal $l$ generated by $\hat{\tilde{\mu}}$ in $\operatorname{Exp}\left(\mathbb{C}^{n}\right)$ is slowly decreasing in the sense of Berenstein and Taylor $([1])$, we can apply $[1,5.4(i i)]$ (see also [22, Theorem 12]) to conclude that

$$
I=I_{\text {loc }}:=\left\{g \in \operatorname{Exp}\left(\mathbb{C}^{N}\right):[g]_{a} \in I_{a} \text { for all } a \in \mathbb{C}^{N}\right\} .
$$

It follows from the hypothesis that $\hat{v} \in I_{\text {loc }}$. Consequently $\hat{v} \in I$ and $f \in \operatorname{Exp}\left(\mathbb{C}^{N}\right)$.

The Fourier-Laplace transform $F: H\left(\mathbb{C}^{N}\right)^{\prime} \rightarrow \operatorname{Exp}\left(\mathbb{C}^{N}\right), F(L)(z):=\left\langle L_{\omega}, e^{-i\langle z, \omega\rangle}\right\rangle$, is an isomorphism $([16,4.5 .3])$. Accordingly we can find $L \in H\left(\mathbb{C}^{N}\right)^{\prime}$ with $F(L)=f$. From this it follows that $\left\langle v,\left.g\right|_{\mathbb{R}}\right\rangle=\langle L, \mu * g\rangle$ for every $g \in H\left(\mathbb{C}^{N}\right)$, where $\mu * g$ denotes the entire function $(\mu * g)(z):=\left\langle\mu_{y}, g(z-y)\right\rangle$.

To conclude we fix $u \in \mathscr{E}_{\omega}\left(\mathbb{R}^{N}\right)$ satisfying $\mu * u=0$ and we prove that $\langle v, u\rangle=0$. To do this take a compact set $K \subset \mathbb{C}^{N}$ such that $|\langle L, g\rangle| \leqq C \sup _{z \in K}|g(z)|$ for every $g \in H\left(\mathbb{C}^{N}\right)$ and some positive constant $C$. Fix $\chi \in \mathscr{D}_{\omega}\left(\mathbb{R}^{N}\right)$ such that $\chi=1$ on a convex neighbourhood of $B(0, R)+\operatorname{supp} \mu+\operatorname{supp} v$, with $R>0$ satisfying $R>\delta+\frac{N}{2}$ and $K \subset B(0, \delta)$. We put $E_{j}(z):=\left(\frac{j}{\pi}\right)^{N / 2} \exp (-j\langle z, z\rangle), v:=\chi u$, and we define

$$
v_{j}(z):=\int_{\mathbb{R}^{N}} v(x) E_{j}(z-x) d x, \quad z \in \mathbb{C}^{N}, \quad j \in \mathbb{N}
$$


(compare with $[\mathbf{1 7}, 9.1 .2])$. An easy calculation yields

$$
\langle v, u\rangle=\langle v, v\rangle=\lim _{j}\left\langle v,\left.v_{j}\right|_{\mathbb{R}^{*}}\right\rangle=\lim _{j}\left\langle L, \mu * v_{j}\right\rangle=0
$$

the last identity being a consequence of the fact that $\mu * v_{j}$ converges to 0 uniformly on $K$.

Corollary. Let $\omega$ be a weight and $\mu \in \mathscr{E}_{\omega}^{\prime}\left(\mathbb{R}^{N}\right), \mu \neq 0$. The set $E_{\mu}$, of all exponential solutions of the equation $\mu * u=0$, is dense in $\operatorname{Ker} T_{\mu}$ (with the topology induced by $\left.\mathscr{E}_{\omega}\left(\mathbb{R}^{N}\right)\right)$.

REMARK 1. Assume $N=1$. Let $\omega$ be a weight and let $\mu \in \mathscr{E}_{\omega}^{\prime}(\mathbb{R}), \mu \neq 0$. Then $T_{\mu}^{t}\left(\mathscr{E}_{\omega}^{\prime}(\mathbb{R})\right)$ is sequentially dense in $\overline{T_{\mu}^{\prime}\left(\mathscr{E}_{\mu}^{\prime}(\mathbb{R})\right)}$.

Proof. In fact, we put $p(z)=\omega(|z|)+|\operatorname{Im} z|$ and we identify, via Fourier-Laplace transform, $\mathscr{E}_{\omega}^{\prime}(\mathbb{R})$ with $A_{p}, T_{\mu}^{\prime}$ with the multiplication operator $A_{p} \rightarrow A_{p}, f \rightarrow \widehat{\tilde{\mu} f}$, and $T_{\mu}^{\prime}\left(\mathscr{E}_{\omega}^{\prime}(\mathbb{R})\right)$ with the principal ideal $I$ generated by $\hat{\hat{\mu}}$ in $A_{p}$. We apply $[19,6.13]$ to get that $p$ is stable $([19$, Definition 6.3$])$. Now we deduce from the proof of $[19,6.6,6.7]$ that $I$ is sequentially dense in $\bar{l}$.

Real-analytic functions. Let $K$ be a compact set in $\mathbb{R}^{N}$ and let $\left(V_{n}\right)$ be a fundamental (decreasing) sequence of open neighbourhoods of $K$ in $\mathbb{C}^{N}$. Then $A(K):=$ ind $H^{\infty}\left(V_{n}\right)$ is the space of germs of holomorphic functions on $K$. Given two compact sets $K_{1} \subset K_{2}$ in $\mathbb{R}^{N}$, it follows from $[17,9.1 .2]$ that the restriction map $A\left(K_{2}\right) \rightarrow A\left(K_{1}\right)$ has dense range. The space of real-analytic functions is defined by $A\left(\mathbb{R}^{n}\right):=\underset{K \subset \mathbb{R}^{N}}{\operatorname{proj}} A(K)$ and it is a (reduced) projective limit of complete (LB)-spaces. We collect in the next proposition some of the known properties of $A\left(\mathbb{R}^{N}\right)$ which will be needed later.

Proposition 2. Let $\omega$ be a weight and $\mu \in \mathscr{E}_{\omega}^{\prime}\left(\mathbb{R}^{N}\right), \mu \neq 0$. Then:

(1) $A\left(\mathbb{R}^{N}\right)$ is contained in $\mathscr{E}_{\omega}\left(\mathbb{R}^{N}\right)$ and the inclusion $A\left(\mathbb{R}^{N}\right) \rightarrow \mathscr{E}_{\omega}\left(\mathbb{R}^{N}\right)$ is continuous.

(2) The convolution operator $T_{\mu}: A\left(\mathbb{R}^{N}\right) \rightarrow A\left(\mathbb{R}^{N}\right)$ is well-defined and continuous.

(3) An entire function $f \in H\left(\mathbb{C}^{N}\right)$ is the Fourier-Laplace transform of some functional $L \in\left(A\left(\mathbb{R}^{N}\right)\right)^{\prime}$ if and only if

$$
\exists A>0 \quad \forall \epsilon>0 \quad \exists C_{\epsilon}>0:|f(z)| \leq C_{\epsilon} \exp (A|\operatorname{Im} z|+\epsilon|z|) .
$$

We only make some remarks about the proofs. Concerning property (1) observe that, given a compact set $K \subset \mathbb{R}^{N}$ and an open set $V$ in $\mathbb{C}^{N}, K \subset V$, there is $C>0$ such that for every $f \in A\left(\mathbb{R}^{N}\right)$ with holomorphic and bounded extension to $V$ (denoted also by $f$ ) we have

$$
\left|f^{(\alpha)}(x)\right| \leq C^{|\alpha|+1}(|\alpha| !) \sup _{z \in V}|f(z)|
$$

Since $\omega(t)=o(t)$ as $t$ tends to infinity, for each $m \in \mathbb{N}$ there is $B_{m}>0$ with

$$
C^{|\alpha|+1}(|\alpha| !) \leq B_{m} \exp \left(m \varphi^{*}\left(\frac{|\alpha|}{m}\right)\right) \leq B_{m} \sup _{z \in V}|f(z)| .
$$


For a proof of (3) see $[18,15.1 .5]$, while (2) is a consequence of $[\mathbf{1 7}, 9.1 .2]$.

Remarks 2. (a) Property (1) implies that the restriction map $H\left(\mathbb{C}^{N}\right) \rightarrow \mathscr{E}_{\omega}\left(\mathbb{R}^{N}\right)$ is a continuous linear map, since it has closed graph.

(b) It follows from (3) that we can define an $(L F)$ topology in the dual of $A\left(\mathbb{R}^{N}\right)$ which is finer than the weak topology $\sigma\left(A\left(\mathbb{R}^{N}\right)^{\prime}, A\left(\mathbb{R}^{N}\right)\right)$.

The next lemma will be applied for $E:=\mathscr{E}_{\omega}\left(\mathbb{R}^{N}\right), T:=T_{\mu}$ and $F:=A\left(\mathbb{R}^{N}\right)$.

Lemma 1. Let E be a Fréchet-Schwartz space and let $F$ be a locally convex space such that there is a continuous inclusion $j: F \rightarrow E$ with dense range. Assume $F^{\prime}$ admits an $(L F)$ topology $\tau$ finer than the weak topology $\sigma\left(F^{\prime}, F\right)$. Let $T \in L(E, E)$ be a continuous linear operator such that $T(F) \subset F$. If

$$
\forall u \in \overline{T^{\prime} E^{\prime}} E_{b} \quad \exists ! v \in F^{\prime}:\langle v, T f\rangle=\left\langle j^{t} u, f\right\rangle,
$$

then $F$ is contained in $T(E)$.

Proof. We denote by $G$ the space $\overline{T^{\prime} E^{\prime}} E_{b}^{\prime} \subset E_{b}^{\prime}$, always endowed with the induced topology. It is well known that $G$ is (also) a (DFS)-space. First define $R: G \rightarrow F^{\prime}$ by $R u:=v$, where $v \in F^{\prime}$ is the unique element given by $(*)$. Certainly $R$ is well-defined, linear and satisfies $R T^{\prime}=j^{\prime}$ on $E^{\prime}$. Moreover $R: G \rightarrow\left(F^{\prime}, \tau\right)$ has closed graph, hence it is continuous. To complete the proof we take $f \in F$ and we define $\Phi: G \rightarrow \mathbb{C}$ by $\Phi(u):=\langle f, R u\rangle$. Clearly $\Phi$ is continuous on $G$. By the Hahn-Banach theorem (and the reflexivity of $E$ ), there is $g \in E$ such that the restriction of $g$ to $T^{\prime} E^{\prime}$ coincides with $\Phi$. This implies $T g=j f$. Indeed, for each $u \in E^{\prime}$ we have

$$
\langle T g, u\rangle=\left\langle g, T^{t} u\right\rangle=\Phi\left(T^{t} u\right)=f\left(R T^{t} u\right)=f\left(j^{t} u\right)=\langle j f, u\rangle
$$

To prepare the proof of the next theorem, we recall that if $\mu \in \mathscr{E}_{\omega}^{\prime}\left(\mathbb{R}^{N}\right)$ there is a compact set $K$ in $\mathbb{R}^{N}$ such that, for some $m \in \mathbb{N}$ and $C>0,|\langle\mu, f\rangle| \leq C P_{K, m}(f)$ for every $f \in \mathscr{E}_{\omega}\left(\mathbb{R}^{N}\right)$. Accordingly $\langle\mu, f\rangle=0$ for every $f \in \mathscr{D}_{\omega}\left(\mathbb{R}^{N}\right)$ with $\operatorname{supp} f \cap K=\varnothing$. The smallest compact set $K$ in $\mathbb{R}^{N}$ with the above property is called the support of $\mu$. The classical theorem of supports remains true for arbitrary $\mu, v \in \mathscr{E}_{\omega}^{\prime}\left(\mathbb{R}^{N}\right)$, i.e.,

$$
\operatorname{conv}(\operatorname{supp}(\mu * v))=\operatorname{conv}(\operatorname{supp} \mu)+\operatorname{conv}(\operatorname{supp} v) \text {. }
$$

THEOREM 1. For every $\mu \in \mathscr{E}_{\omega}^{\prime}\left(\mathbb{R}^{N}\right), \mu \neq 0$, the space of real-analytic functions $A\left(\mathbb{R}^{N}\right)$ is contained in the range of the convolution operator $T_{\mu}: \mathscr{E}_{\omega}\left(\mathbb{R}^{N}\right) \rightarrow \mathscr{E}_{\omega}\left(\mathbb{R}^{N}\right)$.

Proof. We check condition (*) in Lemma 1. In view of Proposition 1 , it is enough to show that, given $v \in \mathscr{E}_{\omega}^{\prime}\left(\mathbb{R}^{N}\right)$ such that $f:=\hat{v} / \hat{\tilde{\mu}}$ is an entire function on $\mathbb{C}^{N}$, the function $f$ belongs to $A\left(\mathbb{R}^{N}\right)^{\prime}$. To see that, since the theorem of supports holds, we can adapt the proof of $[\mathbf{1 8}, 16.4 .3]$ (also see $[\mathbf{1 8}, 16.2 .1$ and 16.3.1]) to get

$$
\exists A>0 \quad \forall \epsilon>0 \quad \exists C_{\epsilon}>0:|f(z)| \leq C_{\epsilon} \exp (A|\operatorname{Im} z|+\epsilon|z|) .
$$

The conclusion follows from Proposition 2(3) and Lemma 1.

\section{Non-quasianalytic functions.}

Lemma 2. Let $E$ and $F$ be Fréchet-Schwartz spaces such that there is a continuous inclusion $j: F \rightarrow E$ with dense range. Let $T \in L(E, E)$ be such that $T(E)$ is dense in $E$, 
$T(F) \subset F$ and the restriction $S$ of $T$ to $F$ belongs to $L(F, F)$ and such that it has also dense range. If we have $E_{b}^{\prime}=\operatorname{ind}\left(G_{n},\|\cdot\|_{n}\right)$ and $F_{b}^{\prime}=\operatorname{ind}\left(H_{k},||_{k}\right)$, the following conditions are equivalent:

(1) $F$ is contained in $T(E)$

(2) $j^{t}\left(T^{t}\right)^{-1}: T^{\prime} E^{\prime} \subset E_{h}^{\prime} \rightarrow F_{b}^{\prime}$ is continuous

(3) $\forall u \in \overline{T^{\prime} E^{\prime}} E_{b}^{\prime} \exists ! v \in F^{\prime}: S^{t} v=j^{\prime} u$. These three equivalent conditions imply

(4) $\forall n \quad \exists k, C>0 \forall u \in E^{\prime}:\left|j^{\prime} u\right|_{k} \leq C\left\|T^{\prime} u\right\|_{n}$. If $T^{\prime} E^{\prime}$ is bornological for the topology induced by $E_{b}^{\prime}$, then (4) and (1) are equivalent.

Proof. First observe that $T^{\prime}, S^{\prime}$ and $j^{\prime}$ are injective. The equivalence of (1) and (2) is a particular case of Proposition 2.1 in [2]. To prove (2) implies (3) consider the unique continuous linear extension $R: \overline{T^{\prime} E^{\prime} E_{h}} \rightarrow F_{b}^{\prime}$ of $j^{\prime}\left(T^{t}\right)^{-1}$. Given $u \in \overline{T^{\prime} E^{\prime} E_{b}}$ put $v:=$ $R u \in F^{\prime}$. Then $S^{\prime} v=j^{\prime} u$, which proves condition (3). The uniqueness follows from the injectivity of $S^{t}$. Since (3) implies condition (*) in Lemma 1, (3) implies (1).

To complete the proof it is enough to observe that (4) means exactly that the linear map $j^{\prime}\left(T^{\prime}\right)^{-1} ; T^{\prime} E^{\prime} \subset E_{b}^{\prime} \rightarrow F_{b}^{\prime}$ maps bounded sets into bounded sets.

In general (4) does not imply (1), as was shown in $[2,2.12]$.

To state our main result, if $\omega \leq \sigma$ are two weights, we put $p(z):=\omega(|z|)+|\operatorname{Im} z|$, $q(z):=\sigma(|z|)+|\operatorname{Im} z|, z \in \mathbb{C}^{N}$. We identify, via Fourier-Laplace transform, $\mathscr{E}_{\omega}^{\prime}\left(\mathbb{R}^{N}\right)=A_{p}$ and $\mathscr{E}_{r}^{\prime}\left(\mathbb{R}^{N}\right)=A_{q}$.

In the applications of Lemma $2, E:=\mathscr{E}_{\omega}\left(\mathbb{R}^{N}\right), F:=\mathscr{E}_{r r}\left(\mathbb{R}^{N}\right), T:=T_{\mu}$ for $\mu \in \mathscr{E}_{\omega}^{\prime}\left(\mathbb{R}^{N}\right)$. In this case,

(1) is equivalent to $\mathscr{E}_{\sigma}\left(\mathbb{R}^{N}\right) \subset T_{\mu}\left(\mathscr{E}_{\omega}\left(\mathbb{R}^{N}\right)\right)$.

(3) is equivalent to the following condition:

$$
\text { if } u \in A_{p} \text { satisfies } f:=u / \hat{\mu} \in H\left(\mathbb{C}^{N}\right) \text { then } f \in A_{q} .
$$

This follows from Proposition 1.

(4) means that for every $B \subset A_{p}$ such that $T_{\mu}^{\prime}(B)$ is bounded in $A_{p}$, it follow's that $B$ is bounded in $A_{q}$.

THEOREM 2. The following conditions are equivalent for $\mu \in \mathscr{E}_{\omega}^{\prime}\left(\mathbb{R}^{N}\right), \mu \neq 0$ :

(1) $\mathscr{E}_{c r}\left(\mathbb{R}^{N}\right)$ is contained in $T_{\mu}\left(\mathscr{E}_{\omega}\left(\mathbb{R}^{N}\right)\right)$,

(2) If $u \in A_{p}$ satisfies $f:=u / \hat{\tilde{\mu}} \in H\left(\mathbb{C}^{N}\right)$, then $f \in A_{q}$,

(3) $\forall n \exists k, C>0 \forall f \in A_{p}$ :

$$
\sup _{z \in \mathbb{C}^{N}}|f(z)| \exp (-k|\operatorname{Im} z|-k \sigma(|z|)) \leq C \sup _{z \in \mathbb{C}^{N}}|\hat{\tilde{\mu}}(z) f(z)| \exp (-n|\operatorname{Im} z|-n \omega(|z|)),
$$

(4) $\mu$ is slowly decreasing for $\sigma$, i.e., there is $C>0$ such that for each $x \in \mathbb{R}^{N},|x| \geq C$, there exists $\xi \in \mathbb{C}^{N}$ with $|x-\xi| \leq C \sigma(x)$ and $|\hat{\tilde{\mu}}(\xi)| \geq \exp (-C q(\xi))$,

(5) $T_{\mu}: \mathscr{E}_{\sigma r}\left(\mathbb{R}^{N}\right) \rightarrow \mathscr{E}_{\sigma}\left(\mathbb{R}^{N}\right)$ is surjective,

(6) $u \in A_{q}$ and $f:=u / \hat{\tilde{\mu}} \in H\left(\mathbb{C}^{N}\right)$ imply $f \in A_{q}$.

Proof. Theorem 1 ensures that the hypotheses of Lemma 2 are satisfied. The equivalence of (1) and (2) and of (5) and (6) follow from Lemma 2 by our remarks 
above. It is also a direct consequence of this lemma that (2) implies (3), using the Fourier-Laplace transform. We show that (3) implies (4). The proof is based on ideas of [25]. Condition (3) means that the division operator

$$
\widehat{\tilde{\mu}} A_{p} \subset A_{p} \rightarrow A_{q}, \quad \hat{\tilde{\mu}} f \rightarrow f
$$

maps bounded sets into bounded sets. We write $q_{1}(z):=\sigma(|\operatorname{Re} z|)+|\operatorname{Im} z|, z \in \mathbb{C}^{N}$, and we suppose that (4) is not satisfied. Accordingly we can find a sequence $\left(x_{j}\right)$ in $\mathbb{R}^{N}$ with $\lim \left|x_{j}\right|=\propto$ and such that for each $j \in \mathbb{N}$ and each $\xi \in \mathbb{C}^{N}$ with $\left|\xi-x_{j}\right| \leq C j \sigma\left(x_{j}\right)$, we have $|\overrightarrow{\hat{\mu}}(\xi)| \leq \exp \left(-C j q_{1}(\xi)\right)$, for $C \geq 2 K$, where $K>0$ is the positive constant satisfying $\sigma(2 t) \leq K \sigma(t)$ for all $t>0$ large enough.

For each $j \in \mathbb{N}$ and $R>0$ we consider the function $h_{j . R}: \mathbb{C}^{N} \rightarrow \mathbb{R}$, which equals $|\operatorname{Im} z|$ if $\left|z-x_{j}\right| \geq R$ and with

$h_{j, R}(z):=\sup \left\{u(z): u\right.$ is plurisubharmonic on $B\left(x_{j}, R\right), \lim _{w \rightarrow \xi} \sup u(w) \leq|\operatorname{Im} \xi|$

for every $\xi$ with $\left.\left|\xi-x_{j}\right|=R\right\}$

for $\left|z-x_{j}\right|<R$. By [27], $h_{j, R}$ is plurisubharmonic and continuous on $\mathbb{C}^{N}$ and satisfies $h_{j, R}\left(x_{j}\right) \geq \frac{2 R}{\pi \sqrt{N}}$.

Put $R_{j}:=j \sigma\left(x_{j}\right), h_{j}:=h_{j, R_{j}}$ and define $\varphi_{j}$ as the function $\varphi_{j}(z):=h_{j, R_{j}+1}(z)+1$. Clearly $\varphi_{j}$ is also plurisubharmonic.

As in $[\mathbf{2 6}, 1.8]$ we can apply $[\mathbf{1 6}, 4.4 .4]$ to find $f_{j} \in H\left(\mathbb{C}^{N}\right)$ with

$$
\left|f_{j}\left(x_{j}\right)\right| \geq \exp \left(\inf _{\left|\omega-x_{j}\right| \leq 1} \varphi_{j}(\omega)-C_{N} \log \left(1+\left|x_{j}\right|\right)^{2}\right)
$$

and

$$
\left|f_{j}(z)\right| \leq C_{N} \exp \left(\sup _{|\omega-z| \leq 1} \varphi_{j}(\omega)+C_{N} \log \left(1+|z|^{2}\right)\right),
$$

where $C_{N}$ is a positive constant which only depends on $N$.

For $\xi \in \mathbb{C}^{N}$ with $\left|\xi-x_{j}\right| \geq R_{j}+2$ we have

$$
\sup _{|\omega-\xi| \leq 1} \varphi_{j}(\omega)=\sup _{|\omega-\xi| \leq 1}|\operatorname{Im} \omega|+1 \leq|\operatorname{Im} \xi|+2
$$

and

$$
\left|f_{j}(\xi)\right| \leq C_{N^{\prime}} \exp \left(|\operatorname{Im} \xi|+2+C_{N} \log \left(1+|\xi|^{2}\right)\right),
$$

which implies $f_{j} \in A_{p}$. In fact it is even the Fourier-Laplace transform of some distribution with compact support.

We show that $\left(\hat{\tilde{\mu}} f_{j}\right)$ is bounded in $A_{p}$ but $\left(f_{j}\right)$ is not bounded in $A_{q}$. This contradicts (3). Since $\lim _{|x| \rightarrow \infty} \frac{\sigma(|x|)}{|x|}=0$, there is $j_{0} \in \mathbb{N}$ such that, for $j \geq j_{0}, R_{j}+2 \leq 2 R_{j} \leq \frac{\left|x_{j}\right|}{2}$. If $j \geq j_{0}$ and $\xi \in \mathbb{C}^{N}$ satisfies $\left|\xi-x_{j}\right|<R_{j}+2$, we get

$$
\sigma\left(x_{j}\right) \leq \sigma(2|\operatorname{Re} \xi|) \leq K \sigma(\operatorname{Re} \xi) \leq K q_{1}(\xi)
$$


and

$$
\sup _{|\omega-\xi| \leq 1} \varphi_{j}(\omega) \leq 2 j \sigma\left(x_{j}\right)+5 \leq 2 K j q_{1}(\xi)+5
$$

This implies, for $j \geq j_{0}$ and $\left|\xi-x_{j}\right|<R_{j}+2$,

$$
\left|f_{j}(\xi)\right| \leq C_{N^{\prime}} \exp \left(2 K j q_{1}(\xi)+5+C_{N} \log \left(1+|\xi|^{2}\right)\right) .
$$

If $j \geq j_{0}$ and $\left|\xi-x_{j}\right|<R_{j}+2 \leq C R_{j}$, we have

$$
\begin{aligned}
\left|\hat{\tilde{\mu}}(\xi) f_{j}(\xi)\right| & \leq C_{N} \exp \left(2 K j q_{1}(\xi)-C_{j q_{1}}(\xi)+5+C_{N} \log \left(1+|\xi|^{2}\right)\right) \\
& \leq C_{N} \exp \left(5+C_{N} \log \left(1+|\xi|^{2}\right)\right) .
\end{aligned}
$$

For $\xi \in \mathbb{C}^{N}$ with $\left|\xi-x_{j}\right| \geq R_{j}+2$ we get

$$
\left|\hat{\tilde{\mu}}(\xi) f_{j}(\xi)\right| \leq|\hat{\mu}(\xi)| C_{N} \exp \left(|\operatorname{Im} \xi|+2+C_{N} \log \left(1+|\xi|^{2}\right)\right) .
$$

Since $\hat{\tilde{\mu}} \in A_{p}$ and $\log \left(1+|\xi|^{2}\right)=O(\omega(|\xi|))$ as $|\xi|$ tends to infinity we conclude

$$
\left|\hat{\tilde{\mu}}(\xi) f_{j}(\xi)\right| \leq D C_{N} \exp (D+(K+m) \omega(|\xi|)+(m+1)|\operatorname{Im} \xi|)
$$

for some constant $D>0$ and some $k \in \mathbb{N}$. Thus there is $L \in \mathbb{N}$ with

$$
\sup _{j \geq j_{0}} \sup _{\xi \in \mathbb{C}^{N}}\left|\hat{\mu}(\xi) f_{j}(\xi)\right| \exp (-L p(\xi))<\infty .
$$

On the other hand, it is easy to show that $\sup _{|\omega-z| \leq 1} h_{j}(\omega) \leq \varphi_{j}(z)$ for all $\left|z-x_{j}\right| \leq R_{j}+1$, hence $\inf _{\left|z-x_{j}\right| \leq 1} \varphi_{j}(z) \geq h_{j}\left(x_{j}\right)$, which yields

$$
\left|f_{j}\left(x_{j}\right)\right| \geq \exp \left(h_{j}\left(x_{j}\right)-C_{N} \log \left(1+\left|x_{j}\right|^{2}\right)\right) \geq \exp \left(\frac{2 j \sigma\left(x_{j}\right)}{\pi \sqrt{N}}-C_{N} \log \left(1+\left|x_{j}\right|^{2}\right)\right) .
$$

This implies $\sup _{j \geq j_{0}}\left|f_{j}\left(x_{j}\right)\right| \exp \left(-s q\left(x_{j}\right)\right)=\infty$ for all $s \in \mathbb{N}$.

Since (5) clearly implies (1), we complete the proof indicating how to show that (4) implies (5). Let $u \in A_{q}$ satisfy that $f:=u / \hat{\tilde{\mu}}$ is an entire function on $\mathbb{C}^{N}$. Since $u$ and $\tilde{\tilde{\mu}}$ belong to $A_{q}$ there is $A>0$ such that

$$
\max (|u(\xi)|,|\hat{\tilde{\mu}}(\xi)|) \leq A \exp (A q(\xi)), \quad \xi \in \mathbb{C}^{N} .
$$

Fix $x \in \mathbb{R}^{N},|x| \geq C$, and put $r:=C \sigma(x)$. We apply $[15,3.2]$ to get

$$
|f(x)| \leq \frac{\left(\sup _{|x-\xi|<4 r}|u(\xi)|\right)\left(\sup _{|x-\xi|<4 r}|\hat{\mu}(\xi)|\right)}{\left(\sup _{|x-\xi|<r}|\hat{\tilde{\mu}}(\xi)|\right)^{2}}
$$

from where it follows, on account of (4), that $|f(x)| \leq D \exp (D \sigma(x))$ for all $x \in \mathbb{R}^{N}$ and some $D>0$. Since $f \in \operatorname{Exp}\left(\mathbb{C}^{N}\right)$ (see the proof of Proposition 1), we can apply the Phragmen-Lindelöf principle as in $[23,2.2]$ to conclude that $f \in A_{q}$. 
In particular, restricting to the Gevrey classes we get the following result. $\mu \neq 0$ :

THEOREM 3. Let $1<t<s$. The following conditions are equivalent for $\mu \in \Gamma^{(s)}\left(\mathbb{R}^{N}\right)^{\prime}$,

(1) $\Gamma^{(t)}\left(\mathbb{R}^{N}\right)$ is contained in $T_{\mu}\left(\Gamma^{(s)}\left(\mathbb{R}^{N}\right)\right)$,

(2) $T_{\mu}: \Gamma^{(t)}\left(\mathbb{R}^{N}\right) \rightarrow \Gamma^{(t)}\left(\mathbb{R}^{N}\right)$ is surjective.

REMARK 3. If $\mu \in \mathscr{E}_{\omega}^{\prime}\left(\mathbb{R}^{N}\right)$ one can also define the convolution operator $S_{\mu}: \mathscr{D}_{c r}^{\prime}\left(\mathbb{R}^{N}\right) \rightarrow \mathscr{D}_{c r}^{\prime}\left(\mathbb{R}^{N}\right), S_{\mu}(v):=\mu * v$ with $\langle\mu * v, f\rangle=\langle v, \check{\mu} * f\rangle$ for $f \in \mathscr{D}_{\sigma}\left(\mathbb{R}^{N}\right)$. The equivalent conditions of Theorem 2 are also equivalent to

(7) $S_{\mu}$ has a fundamental solution in $\mathscr{D}_{r}^{\prime}$.

(See for instance [12] and [11, 6.4]).

It follows from the remark above that a surjective convolution operator on a Beurling class $\mathscr{C}_{\omega}\left(\mathbb{R}^{N}\right)$ is also surjective on a smaller class $\mathscr{E}_{\sigma}\left(\mathbb{R}^{N}\right), \omega \leq \sigma$.

EXAMPLE. There are weights $\omega \leq \sigma$ and $\mu \in \mathscr{E}^{\prime}\left(\mathbb{R}^{N}\right)$ such that $T_{\mu}: \mathscr{E}_{\sigma}\left(\mathbb{R}^{N}\right) \rightarrow \mathscr{E}_{\sigma}\left(\mathbb{R}^{N}\right)$ is surjective but $T_{\mu}: \mathscr{E}_{\omega}\left(\mathbb{R}^{N}\right) \rightarrow \mathscr{E}_{\omega}\left(\mathbb{R}^{N}\right)$ is not surjective.

In fact, let us consider two weights $\omega \leq \sigma$ satisfying properties $\left(\alpha^{\prime}\right),(\beta),(\gamma),(\delta)$. Assume there is some function $m:[0, \infty[\rightarrow[0, \infty[$ such that $\omega(t)=o(m(t))$ and $m(t)=$ $o(\sigma(t))$ as $t$ tends to infinity. We apply [13, Theorem $\left.4^{\prime}\right]$ to find $\mu_{1} \in \mathscr{E}^{\prime}(\mathbb{R})$ such that $\mu_{1}$ is slowly decreasing with respect to $\sigma$ and there is a sequence $\left(t_{k}\right)$ of zeros of $\widehat{\mu_{1}}$ with order $o\left(T_{k}, \widehat{\mu_{1}}\right) \geq m\left(t_{k}\right)$. Since $\lim _{t \rightarrow \infty} \frac{o\left(T_{k}, \widehat{\mu_{1}}\right)}{\omega\left(t_{k}\right)}=\propto$ we conclude that $\mu_{1}$ is not slowly decreasing with respect to $\omega\left(\left[14\right.\right.$, Theorem 5]). Finally we define $\mu \in \mathscr{E}^{\prime}\left(\mathbb{R}^{N}\right)$ by $\hat{\mu}(z)=\widehat{\mu_{1}}\left(z_{1}\right) \ldots$ $\widehat{\mu_{1}}\left(z_{N}\right), z=\left(z_{1}, \ldots, z_{N}\right) \in \mathbb{C}^{N}$.

Ehrenpreis $[9,6.2]$ constructed a distribution $\mu \in \mathscr{E}^{\prime}(\mathbb{R})$ such that the convolution operator $T_{\mu}$ is not surjective on every non-quasianalytic class $\mathscr{E}_{\omega}(\mathbb{R})$.

Ehrenpreis proved in $[9,2.4]$ that $T_{\mu}^{t}\left(\mathscr{E}^{\prime}\left(\mathbb{R}^{N}\right)\right)$ is bornological (with the topology induced by $\mathscr{E}^{\prime}\left(\mathbb{R}^{N}\right)$ ) for every invertible distribution $\mu \in \mathscr{E}^{\prime}\left(\mathbb{R}^{N}\right)$ (i.e. slowly decreasing with respect to $\omega(t)=\log (1+t))$ and asked whether the hypothesis on the invertibility of $\mu$ can be removed or not. Since every sequentially dense subspace of a (DFS)-space is bornological $([2,2.4])$, it follows from our Remark 1 (and the known fact that a closed subspace of a (DFS)-space is also a (DFS)-space) that, in case $N=1, T_{\mu}^{\prime}\left(\mathscr{E}^{\prime}(\mathbb{R})\right)$ is bornological for every distribution $\mu \in \mathscr{E}^{\prime}(\mathbb{R})$.

Proposition 3. Let $\omega$ be a weight and $\mu \in \mathscr{E}_{\omega}^{\prime}\left(\mathbb{R}^{N}\right), \mu \neq 0$. Assume $\mu$ is slowly decreasing for some weight $\sigma, \sigma \geq \omega$. Then $T_{\mu}^{t}\left(\mathscr{E}_{\omega}^{\prime}\left(\mathbb{R}^{N}\right)\right)$ is bornological. In particular there are non-invertible distributions $\mu \in \mathscr{E}^{\prime}\left(\mathbb{R}^{N}\right)$ such that $T_{\mu}^{\prime}\left(\mathscr{C}^{\prime}\left(\mathbb{R}^{N}\right)\right)$ is bornological.

Proof. We only have to prove that $T_{\mu}^{t}\left(\mathscr{E}_{\omega}^{\prime}\left(\mathbb{R}^{N}\right)\right)$ is sequentially dense in $\overline{T_{\mu}^{\prime}\left(\mathscr{E}_{\omega}^{\prime}\left(\mathbb{R}^{N}\right)\right)}$. To do this, fix $v \in \overline{T_{\mu}^{t}\left(\mathscr{E}_{\omega}^{\prime}\left(\mathbb{R}^{N}\right)\right)}$ and apply Proposition 1 and Theorem 2 to find $v_{1} \in \mathscr{E}_{\omega}^{\prime}\left(\mathbb{R}^{N}\right)$ such that $\hat{v} / \hat{\vec{\mu}}=\widehat{v_{1}}$. Hence $v=\check{\mu} * v_{1}$ in $\mathscr{E}_{\omega}^{\prime}\left(\mathbb{R}^{N}\right)$. We take a sequence $\left(\phi_{n}\right) \subset \mathscr{D}_{r}\left(\mathbb{R}^{N}\right)$ which is a regularising sequence in $\mathscr{C}_{\omega}\left(\mathbb{R}^{N}\right)$ and apply our hypothesis to find $g_{n} \in \mathscr{C}_{\omega}\left(\mathbb{R}^{N}\right)$ satisfying $\check{\mu} * g_{n}=\check{\phi}_{n}$. Then it is easy to get $v * g_{n} \in \mathscr{D}_{\omega}\left(\mathbb{R}^{N}\right), v * \check{\phi}_{n}=$ $T_{\mu}^{\prime}\left(v * g_{n}\right)$ and $v=\lim _{n \rightarrow \infty}\left(v * \check{\phi}_{n}\right)$.

ACKNowledgement. The research was supported by DGICYT under Proyecto PB91-0538. The authors want to express their gratitude to Prof. Reinhold Meise for his 
interest in the present research. In particular he pointed out to us that Momm's articles could be helpful for the problems we were discussing.

\section{REFERENCES}

1. C. A. Berenstein and B. A. Taylor, Interpolation problems in $\mathbb{C}^{N}$ with applications to harmonic analysis. J. d'Analyse Math. 38 (1980), 188-254.

2. J. Bonet, R. Meise and B. A. Taylor, On the range of the Borel map for classes of non-quasianalytic functions, in: Progress in Functional Analysis, North Holland Math. Studies 170 (1992), 97-111.

3. R. W. Braun, R. Meise and B. A. Taylor, Ultradifferentiable functions and Fourier analysis, Resultate Math. 17 (1990), 206-237.

4. R. W. Braun, R. Meise and D. Vogt, Existence of fundamental solutions and surjectivity of convolution operators on classes of ultradifferentiable functions, Proc. London Math. Soc. (3) 61 (1990), 344-370.

5. Ch. Chou, La Transformation de Fourier Complexe et l'Équation de Convolution, Lecture Notes in Math. 325 (Springer Verlag, 1973).

6. I. Cioranescu, Convolution equations in $\omega$-ultradistribution spaces, Rev. Roum. Math. Pures et Appl. 25 (1980), 719-737. $883-903$.

7. L. Ehrenpreis, Solution to some problems of division, Part I. Amer. J. Math. 76 (1954), $685-715$.

8. L. Ehrenpreis, Solution to some problems of division, Part III. Amer. J. Math. 78 (1956),

9. L. Ehrenpreis, Solution of some problems of division, Part IV. Invertible and elliptic operators, Amer. J. Math. 82 (1960), 522-588.

10. L. Ehrenpreis and P. Malliavin, Invertible operators and interpolation in $A U$ spaces, $J$. Math. Pures Appl. 53 (1974), 165-182.

11. K. Floret, Some aspects of the theory of locally convex inductive limits, Functional Analysis: Surveys and Recent Results II, North-Holland Math. Studies 38 (1980), 205-237.

12. O. V. Grudzinski, Konstruktion von Fundamentallösungen für Convolutoren, Manuscripta Math. 19 (1976), 283-317.

13. O. V. Grudzinski, Examples of solvable and non-solvable convolution equations in $K_{p}^{\prime}$, $p \geq 1$, Pacific Journal Math. 80 (1979), 561-574.

14. O. V. Grudzinski, Slowly decreasing entire functions and convolution equations, Partial Differential Equations, Banach Center Publication 10 (1983), 169-184.

15. L. Hörmander, On the range of convolution operators, Ann. of Math. 76 (1962), 148-170.

16. L. Hörmander, An Introduction to Complex Analysis in Several Variables (Princeton University Press, 1967).

17. L. Hörmander, The Analysis of Linear Partial Differential Operators I (Springer, 1983).

18. L. Hörmander, The Analysis of Linear Partial Differential Operators II (Springer, 1983).

19. J. J. Kelleher and B. A. Taylor, Closed ideals in locally convex algebras, J. reine angew. Math. 255 (1972), 190-209.

20. B. Malgrange, Existence et approximation des solutions des équations aux dérivées partielles et des équations de convolution, Ann. Inst. Fourier Grenoble 6 (1955-56), 271-355.

21. B. Malgrange, Sur la propagation de la régularité des solutions des équations a coefficients constants, Bull. Math. Soc. Math. Phys. Roumanie 3 (53) (1959), 443-446.

22. R. Meise and B. A. Taylor, Each non-zero convolution operator on the entire functions admits a continuous linear right inverse, Math. Z. 197 (1988), 139-152.

23. R. Meise and B. A. Taylor, Whitney's extension theorem for ultradifferentiable functions of Beurling type, Arkiv Mat. 26 (1988), 265-287.

24. R. Meise, B. A. Taylor and D. Vogt, Equivalence of slowly decreasing conditions and local Fourier expansion, Indiana Univ. Math. J. 36 (1987), 729-756.

25. S. Momm, Closed ideals in nonradial Hörmander algebras, Archiv Math. 58 (1992), 47-55. 
26. S. Momm, Division problems in spaces of entire functions of finite order, Functional Analysis (Ed. K. D. Bierstedt, A. Pietsch, W. Ruess, D. Vogt) (Marcel Dekker, 1993), 435-457.

27. S. Momm, A Phragmen-Lindelöf theorem for plurisubharmonic functions on cones in $\mathbb{C}^{N}$, Indiana Univ. Math. J. 41 (1992) 861-867.

28. P. Pérez Carreras and J. Bonet, Barrelled Locally Convex Spaces, North-Holland Math. Studies 131 (1987).

Departamento de Matemática Aplicada

E.T.S. ARQUITECTURA

Camino de Vera

E-46071 Valencia, Spain
Departamento de Análisis Matemático UNIVERSIDAD DE VALENCIA

DOCTOR MOLINER 50

E-46100 Burjasot (Valencia), Spain 